\title{
Cost-effectiveness of coronary interventions
}

\author{
David J Cohen, Craig A Sukin
}

As the costs of medical care continue to rise, there has been increasing interest in evaluating the cost-effectiveness of medical practices. One area that has received particular scrutiny in recent years is percutaneous coronary revascularisation (PTCA). In the United States alone, more than 300000 PTCAs are performed each year at an estimated cost of approximately US\$3 billion. Despite the tremendous growth of the PTCA industry, however, there is still no evidence that the procedure improves survival compared with medical treatment, and only one small trial has examined the results of PTCA in a controlled fashion. ${ }^{1}$ Given the cost of these procedures and the limited evidence of their overall effectiveness, it is understandable that PTCA is a prime target for cost containment efforts.

\section{What does cost-effective mean?}

In order to evaluate the cost-effectiveness of percutaneous coronary interventions, it is important to distinguish between "costsaving" and "cost-effective". Although occasionally a new medical treatment is introduced that both improves clinical outcomes and reduces medical care costs, most medical innovations increase the cost of care. Even highly effective treatments such as bypass surgery for left main disease, ${ }^{2} \beta$ blocker therapy after myocardial infarction, ${ }^{3}$ or thrombolytic therapy for acute myocardial infarction, save lives only by increasing medical care costs. ${ }^{4}$ Nonetheless, such treatments are generally viewed as cost-effective because their benefits are "worth the cost".

Cost-effectiveness analysis is a formal technique that is used to maximise the health benefits available to society subject to the constraint of a fixed health care budget. By explicitly quantifying the trade-offs between health care costs and health benefits, costeffectiveness analysis allows physicians to compare the health benefits gained by use of a specific treatment to those benefits that could be achieved by alternative uses of the same health care resources. The appeal of costeffectiveness analysis for medical applications is that health benefits may be measured in any appropriate clinical unit such as lives saved, complications prevented, or years of life gained (rather than assigning monetary value to such outcomes). In the case of PTCA where both quality of life and survival are important outcome measures, health benefits are best measured in terms of quality adjusted life years (QALYs). In this framework, each year of life expectancy is weighted by a utility factor that reflects an individual's preference for his or her state of health relative to perfect health (utility $=1$ ) and death (utility $=0) .{ }^{5}$
Once a programme's net cost and net health benefits have been evaluated, a cost-effectiveness ratio may be calculated as the ratio of these two quantities. Although there is no absolute standard for cost-effectiveness, rough thresholds may be established by comparison with cost-effectiveness ratios for other health care programmes. In general, cost-effectiveness ratios of $<\$ 20000$ per QALY gained (such as those for treatment of severe diastolic hypertension or cholesterol lowering in patients with established coronary heart disease) are viewed as highly favourable. ${ }^{6}$ Incremental cost-effectiveness ratios between $\$ 20000$ and $\$ 40000$ per QALY are also consistent with many accepted medical treatments and may be viewed as reasonably cost-effective. Cost-effectiveness ratios of $>\$ 60000$ to $\$ 80000$ are higher than most generally accepted medical treatments and are thus viewed as relatively unattractive in most medical systems.

\section{PTCA for single vessel disease}

Despite its widespread adoption as an alternative to medical treatment, few studies have specifically addressed the cost-effectiveness of PTCA for patients with stable angina and single vessel coronary disease. In the only randomised trial to date, the ACME investigators found that PTCA improved both anginal symptoms and exercise tolerance compared with standard medical treatment for such patients. ${ }^{1}$ Of patients assigned to medical treatment, $46 \%$ were free from angina at six months' follow up compared with $64 \%$ of those assigned to PTCA. Moreover, patients treated with initial PTCA had a greater improvement in both physical and psychological function than patients assigned to medical therapy. Not surprisingly, medical resource utilisation was also significantly greater with the PTCA strategy. Specifically, PTCA patients were hospitalised for a mean of 3.8 days during the study period compared with 2.4 days for medically treated patients, and $7 \%$ of patients assigned to PTCA required bypass surgery during follow up compared with none of the medically treated patients.

Unfortunately, no formal attempt was made to evaluate the cost-effectiveness of PTCA in the ACME trial. To date, only one study has examined the cost-effectiveness of balloon angioplasty for patients with single vessel coronary disease. ${ }^{7}$ Wong and colleagues developed a detailed computer simulation model to estimate the cost-effectiveness of angioplasty, bypass surgery, and medical treatment for patients with chronic stable angina. They found that compared with medical therapy, angioplasty improved quality adjusted life \\ Interventional \\ Massachusetts, USA \\ C A Sukin \\ Policy and \\ School of Public \\ Health, Boston, \\ D J Cohen \\ Correspondence to: \\ enue, Boston, MA 02215
}


expectancy in all patient subgroups regardless of anginal severity or left ventricular function. Although overall costs were clearly higher with PTCA, it nonetheless appeared to be costeffective compared with medical treatment for most patients with symptomatic single vessel disease. For example, in patients with severe angina, normal ventricular function, and single vessel disease in the left anterior descending (LAD) coronary artery, quality adjusted life expectancy with PTCA as initial therapy was 18.3 QALYs compared with $17 \cdot 4$ QALYs with initial medical treatment, with a highly favourable cost-effectiveness ratio of US\$6000/QALY gained. For patients with mild angina, however, PTCA appeared to be much less cost-effective compared with medical treatment, with an incremental cost-effectiveness ratio of $\$ 80000-\$ 100000 /$ QALY gained. Thus, the available data suggest that for patients with single vessel coronary artery disease and moderate to severe angina, PTCA is reasonably cost-effective compared with medical programmes. As PTCA has not been shown to improve survival, however, treatment of patients with only mild angina or silent ischaemia does not appear to be costeffective.

\section{PTCA for multivessel disease}

In contrast to single vessel disease, there is an abundance of data regarding the cost-effectiveness of PTCA for patients with multivessel disease. Over the past several years, at least five major trials have been published comparing conventional PTCA with bypass surgery for patients with multivessel coronary disease, and economic substudies are available for several of these randomised trials. In general, initial costs with a strategy of PTCA are $30-50 \%$ lower than for bypass surgery, and these cost savings persist for the first year of follow up. Over a three to five year follow up period,

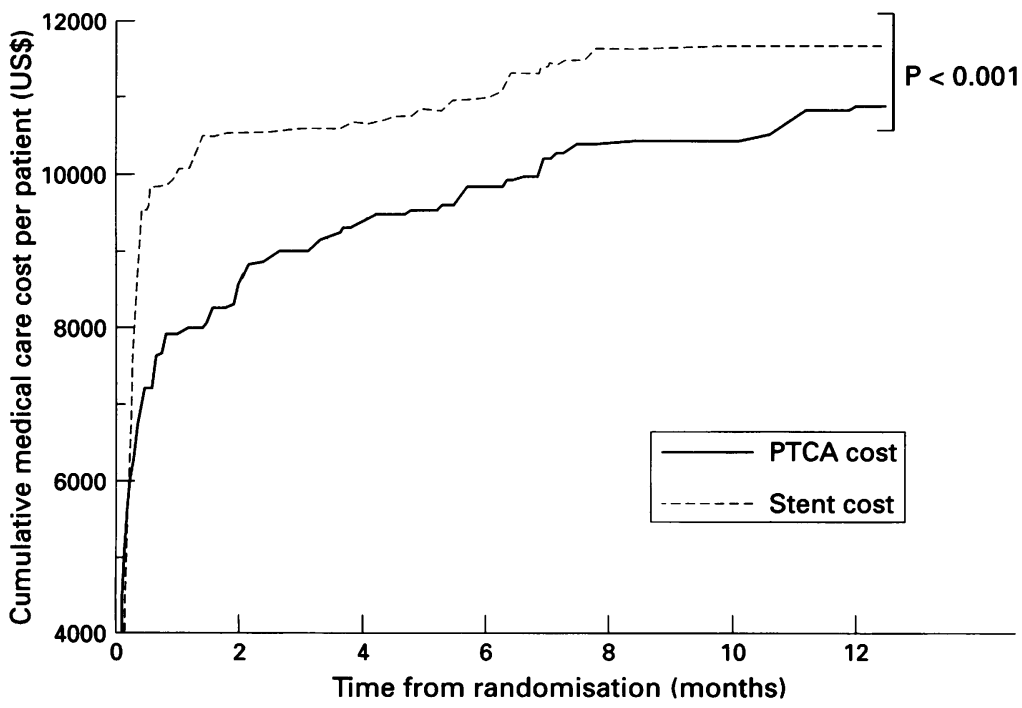

Figure 1 Plot of cumulative medical care costs in patients randomly assigned to initial PTCA $(n=105)$ or coronary stenting $(n=102)$ in the STRESS economic substudy The initial higher cost of stenting was partly offset by $\$ 1400$ in follow up cost savings, but one year costs remained significantly higher with stenting (\$11 656 (5674) v \$10 865 (9073), $P<0.001)$. Adapted from reference 14 with permission. however, much of the initial economic advantage of PTCA is eroded by the need for repeat PTCA or bypass surgery in approximately $50 \%$ of patients. For example, in the Emory angioplasty $v$ surgery trial (EAST) initial costs were $\$ 16200$ with PTCA $v \$ 24000$ with bypass surgery. ${ }^{8}$ By the end of three years of follow up, mean PTCA costs had increased to $93 \%$ of those for bypass surgery. Preliminary results from the Bypass angioplasty revascularization investigation (BARI) were similar with a relative cost difference of only $5 \%$ after five years of follow up. ${ }^{9}$ As survival and late term quality of life were also similar in these studies, it appears that the long term cost-effectiveness of PTCA and bypass surgery are comparable in the management of patients with multivessel disease. Further studies will be needed to identify specific patient subgroups for whom one or the other revascularisation technique is preferred on either clinical or economic grounds.

\section{New coronary interventions}

Over the past decade, a number of new interventional techniques have been developed and introduced into clinical practice including atherectomy devices (directional, rotational, extraction), excimer lasers, and coronary stents. Soon after their introduction, it became clear that hospital costs for these newer procedures were significantly greater than for "plain old balloon angioplasty". ${ }^{10} 11$ As a result, costeffectiveness considerations have played an important role in the evaluation and utilisation of these techniques.

Coronary stenting is the only new device that has been shown to improve angiographic and clinical outcomes compared with balloon angioplasty. 1213 The resulting "stent-mania" has revolutionised the practice of interventional cardiology, but has also placed severe strains on health care reimbursement and hospital budgets in many countries. While it is clear that coronary stenting is associated with improved short and intermediate term outcomes compared with conventional PTCA, current evidence suggests that elective stenting does not pay for itself. In the economic substudy of the randomised STRESS Trial, we found that the in-hospital costs of stenting were $\$ 2200$ higher than for balloon angioplasty ( $\$ 9738 v \$ 7505$ ), yet follow up costs were only $\$ 1400$ lower after stenting (fig 1).14 Thus aggregate one year medical care costs remained approximately $\$ 800$ higher per patient with stenting compared with conventional PTCA

Although recent advances in stent deployment techniques, such as the use of routine high pressure post-dilatation, and the use of combined antiplatelet therapy, have further improved the safety of stenting while shortening length of stay, the net economic impact of stenting remains essentially unchanged. Several studies suggest that optimal stenting without anticoagulation remains significantly more expensive than balloon angioplasty in both the short and long term. ${ }^{15}{ }^{16}$ In a single 
centre study from Boston's Beth Israel Hospital, Sukin and colleagues found that for ideal stent lesions (single, discrete stenoses), optimal stent deployment used $20-25 \%$ more balloons and stents than did stenting in the earlier randomised trials. ${ }^{15}$ Thus, current optimal stenting techniques have increased average catheterisation laboratory costs by nearly $\$ 600$ compared with stenting as performed in the STRESS trial, and by more than $\$ 2200$ compared with conventional PTCA. Thus, even if reduced anticoagulation regimens allow stenting to achieve post-procedure hospital costs identical to those for conventional angioplasty, overall one year costs should remain $\$ 600-\$ 800$ higher with stenting than with PTCA alone.

Nonetheless, it is likely that stenting-at least for discrete coronary stenoses-will eventually become cost saving relative to conventional PTCA. Such cost savings might be achieved through several mechanisms. First, the maturation of the stent market in Europe and the United States should ultimately produce significant reductions in the price of stents. Moreover, development of longer stents and stents without articulation defects should reduce procedural resource consumption as well. Finally, it is possible that optimal stent implantation or second generation stent designs will lead to further reductions in clinical restenosis and thus additional downstream cost savings compared with the STRESS results. These last possibilities are currently the subject of prospective economic evaluations in conjunction with both the Benestent II trial (in Europe) and the STRESS III registry (in the US).

Until such clinical benefits or additional inhospital cost savings are demonstrated, however, the cost-effectiveness of elective coronary stenting depends on whether its proven clinical benefits-namely a reduction in recurrent angina and the need for repeat revascularisation procedures-are sufficient to justify the additional long term costs of the procedure. To evaluate this possibility, we developed a decision-analytic model to study the long term costs and clinical effectiveness of alternative strategies for treating patients with single vessel coronary disease. ${ }^{17} \mathrm{By}$ updating this model to incorporate 1996 cost and outcome data, we estimate that stenting for single vessel coronary disease currently has an incremental costeffectiveness ratio of $\$ 33700 / \mathrm{QALY}$ - similar to the cost-effectiveness of treating mild diastolic hypertension. Thus, the cost-effectiveness of elective coronary stenting appears to compare favourably with other medical practices.

The cost-effectiveness of coronary stenting is sensitive to several key parameters. Figure 2 demonstrates the impact of simultaneous variations in the PTCA abrupt closure and restenosis rates on the cost-effectiveness of elective stenting. If one were willing to spend up to US $\$ 40000 /$ QALY - similar to the costeffectiveness of treating mild hypertensionour model suggests that stenting would be cost-effective as long as the PTCA restenosis

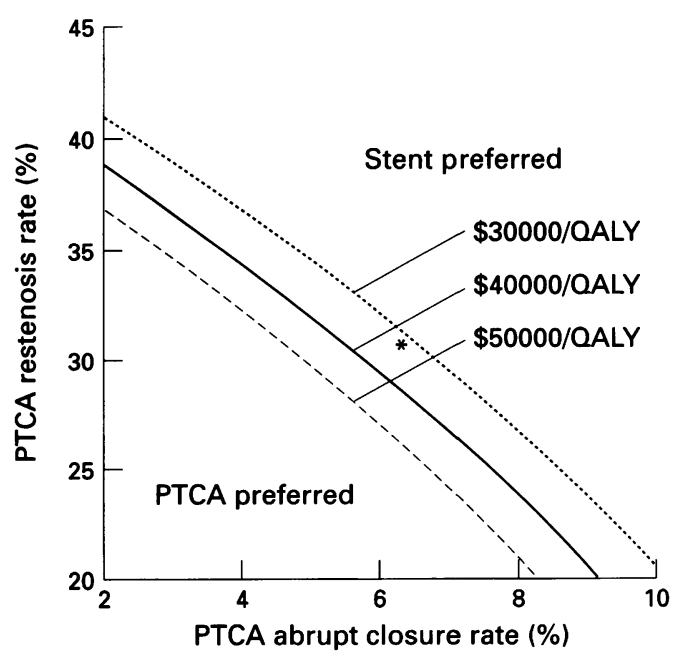

Figure 2 Plot of the relation between angiographic restenosis and abrupt closure rates, the level of acceptable cost-effectiveness, and the optimal revascularisation strategy. For combinations of PTCA abrupt closure and restenosis rates above each threshold line, initial stenting would be preferred at that cost-effectiveness threshold, whereas angioplasty would be preferred for those combinations below the line. The asterix indicates the baseline values for this analysis based on the Benestent and STRESS trials (probability of angiographic restenosis, $31 \%$; probability of abrupt vessel closure, $6 \%$ ).

rate were $>31 \%$ and the PTCA abrupt closure rate were $>5 \%$. On the other hand, if the probabilities of both abrupt closure and restenosis with conventional PTCA were significantly lower than the average results from the randomised clinical trials, then stenting would be significantly less cost-effective. For example, for a type A mid-right coronary stenosis the abrupt closure rate might be only $3 \%$ with an angiographic restenosis rate of $28 \%$. For such a lesion, our model projects a cost-effectiveness ratio for elective stenting of $\$ 200000 /$ QALY - much higher than most accepted medical interventions. These findings suggest that while stenting may be reasonably cost-effective for most suitable lesions, there are other lesions for which PTCA remains the preferred initial treatment.

Most other new coronary devices such as rotational ablation, directional atherectomy, and excimer laser angioplasty are associated with higher procedural and overall hospital costs compared with PTCA as well. ${ }^{18-20}$ In contrast to coronary stenting, however, no other devices have been shown convincingly to improve clinical outcomes compared with PTCA alone. In CAVEAT, directional atherectomy was associated with a somewhat lower rate of angiographic restenosis but there was no difference in six-month clinical outcomes. ${ }^{20}$ Similarly, in the only randomised trial to compare rotational ablation with conventional PTCA, rotational ablation led to a modest decrease in in-hospital complications but no difference in overall outcome at six months. ${ }^{21}$ Given the higher procedural and hospital costs associated with these devices, they do not appear to be cost-effective alternatives to conventional PTCA or stenting for routine angioplasty lesions. 
Conclusions

In today's health care environment, decisions about medical interventions need to reflect measures of cost as well as clinical benefit. While it is clear that PTCA is significantly more expensive than medical therapy alone, analyses incorporating quality of life considerations suggest that angioplasty techniques that have been shown to improve clinical outcomes are, for the most part, cost-effective. For example, by reducing symptoms at a modest cost, balloon angioplasty appears to be reasonably cost-effective compared with medical treatment for patients with moderate to severe angina and single vessel coronary disease. Similarly, coronary stenting increases costs for most patients but is associated with improved outcomes compared with conventional PTCA. Formal cost-effectiveness analysis also suggests that these benefits are worth the cost; at least for patients with discrete stenoses that can be treated with a single stent. On the other hand, most other new devices-including rotational ablation, directional atherectomy, and excimer laser angioplasty-have not been shown to improve clinical outcome compared with balloon angioplasty. Given the higher procedural and hospital costs associated with these devices, it remains difficult to justify their use at present, except for specific lesion subsets for which angioplasty or stenting are unlikely to be successful or in the setting of ongoing clinical investigation.

Dr Cohen was supported in part by a Clinician-Scientist Award from the American Heart Association.

1 Parisi AF, Folland ED, Hartigan P. A comparsion of angioplasty with medical therapy in the treatment of singlevessel coronary artery disease. $N$ Engl $f$ Med 1992;326: 10-16.

2 Weinstein MC, Stason WB. Cost-effectiveness of coronary artery bypass surgery. Circulation 1982;66(suppl III): III56-66.

3 Goldman L, Sia STB, Cook EF, Rutherford JD, Weinstein MC. Costs and effectiveness of routine therapy with long-term beta-adrenergic antagonists after acute long-term beta-adrenergic antagonists after acute
myocardial infarction. $N$ Engl $₹$ Med 1988;319:152-7.

4 Krumholz HM, Pasternak RC, Weinstein MC, Friesinger GC, Ridker PM, Tosteson ANA, et al. Cost effectiveness of thrombolytic therapy with streptokinase in elderly of thrombolytic therapy with streptokinase in elderly patients with suspected acurte

5 Weinstein MC, Stason WB. Foundations of cost-effectiveness analysis for health and medical practices. $N$ Engl $\mathcal{f}$ Med 1977;296:716-21.
6 Goldman L, Gordon DJ, Rifkind BM, Hulley SB, Detsky AS, Goodman DW, et al. Cost and health implications of cholesterol lowering. Circulation 1992;85:1960-8.

7 Wong JB, Sonnenberg FA, Salem DN, Pauker SG. Myocardial revascularization for chronic stable angina. Analysis of the role of percutaneous transluminal coronary angioplasty based on data available in 1989. Ann Intern Med 1990;113:852-71.

8 Weintraub WS, Mauldin PD, Becker E, Kosinski AS, King SB. A comparison of the costs of and quality of life after coronary angioplasty or coronary surgery for multi-vessel coronary artery disease. Results from the Emory Angioplasty Versus Surgery Trial (EAST). Circulation 1995;92:2831-40.

9 Hlatky MA, Boothroyd DB, Winston CA, Brooks MM Mark DB, Johnstone I. Factors affecting long-term cost in multivessel disease patients randomized to PTCA or CABG in the BARI Trial [abstract]. Circulation 1996; 9ABG in

10 Dick RJ, Popma J, Muller DW, Burek KA, Topol EJ. Inhospital costs associated with new percutaneous coronary devices. Am F Cardiol 1991;68:879-85.

11 Cohen DJ, Breall JA, Ho KKL, Weintraub RM, Kuntz RE Weinstein MC, et al. The economics of elective coronary revascularization: comparison of costs and charges for conventional angioplasty, directional atherectomy, stenting, and bypass surgery. 7 Am Coll Cardiol 1993;22. 1052-9.

12 Serruys PW, De Jaegere P, Kiemeneij F, Macay C, Rutsch W, Heyndricks G, et al. A comparison of balloonexpandable-stent implantation with balloon angioplasty in patients with coronary artery disease. $N$ Engl $\mathscr{f} M e d$ in patients with $1994 ; 331: 489-95$.

13 Fischman DL, Leon MB, Baim DS, Schatz RA, Savage MP, Penn I, et al. A randomized comparison of coronarystent placement and balloon angioplasty in the treatment of coronary artery disease. N Engl f $\mathrm{Med}$ 1994;331: 496-501.

14 Cohen DJ, Krumholz HM, Sukin CA, Ho KKL, Siegrist $\mathrm{RB}$, Cleman $\mathrm{M}$, et al. In-hospital and one-year economic outcomes after coronary stenting or balloon angioplasty: results from a randomized clinical trial. Circulation 1995;92:2480-7.

15 Sukin CA, Baim DS, Caputo RP, Ho KKL, Laham RJ, Flatley MG, et al. The impact of optimal stenting techniques on cardiac catheterization laboratory resource utilization and costs. Am $₹$ Cardiol 1997; 79:275-80.

16 Peterson ED, Cowper PA, Zidar JP, Phillips HR, Mark DB. In-hospital costs of coronary stenting (with or without coumadin) compared with angioplasty [abstract] Coumadin) compared

17 Cohen DJ, Breall JA, Ho KKL, Kuntz RE, Goldman L, Baim DS, et al. Evaluating the potential cost-effectiveness of stenting as a treatment for symptomatic singlevessel coronary disease: use of a decision-analytic model. Circulation 1994;89:1859-74

18 Ellis SG, Miller DP, Brown KJ, Omoigui N, Howell GL Kutner M, Topol EJ. In-hospital cost of percutaneous coronary revascularization: critical determinants and implications. Circulation 1995;92:741-7.

19 Guzman LA, Simpfendorfer C, Fix J, Franco I, Whitlow PL. Comparison of costs of new atherectomy devices and balloon angioplasty for coronary artery disease. $A m$ Cardiol 1994;74:22-5.

20 Topol EJ, Leya F, Pinkerton CA, Whitlow PL, Hofling B, Simonton CA, et al. A comparison of directional coronary atherectomy with coronary angioplasty in patients wary atherectomy with coronary angioplasty in patients with coronary artery disease. The

21 Vandormael M, Reifart N, Preusler W, Schwar F, Storger H, Hofman M, et al. Six month follow-up results following excimer laser angioplasty, rotational atherectomy, and balloon angioplasty for complex lesions: ERBAC Study [abstract]. Circulation 1994;90:1213.

\title{
Is a US analysis of cost-effectiveness in interventional cardiology relevant to a centrally funded health care system?
}

\author{
D C Cumberland
}

The University of Sheffield, Clinical Sciences Centre, Northern General Northern General Road, Sheffield S5 7AU, UK
Early studies of costs from the United States were simple comparisons of hospital charges - for example, between coronary bypass and coronary angioplasty. ${ }^{12}$ This generous approach seemed far removed from our centrally funded system in the UK, and we tended to look jealously across the water where costs could be transferred to willing payers on an individual patient basis. Not so now; first, Cohen and Sukin ${ }^{3}$ have done much to clarify the costs, detailing each item of resource consumption and possible cost- 